\title{
3D Simulation of the Closed Shell-Electrode Detector
}

\author{
Ming-Fu FENG ${ }^{a}$ and Zheng $\mathrm{LI}^{\mathrm{b},{ }^{*}}$ \\ ${ }^{1}$ Engineering laboratory of Special Functional thin film Materials, School (Faculty) of Materials \\ Science and Engineering, Xiangtan University, Xiangtan 411105, China.
}
${ }^{2}$ Center for semiconductor Particle and photon Imaging Detector Development and Fabrication, Xiangtan University, Xiangtan 411105, China.

e-mail: amffeng8023@163.com, blizheng@xtu.edu.cn, Tel: +86 1351732860

\begin{abstract}
Keywords: Closed Shell-Electrode Detector (CSED), 3D-Trench electrode detector, device simulation, charge collection property, radiation.
\end{abstract}

\begin{abstract}
A new structure of 3D detectors has been proposed. In order to separate it from the non-etch-through 3D-Trench electrode detectors, we call it as the Closed Shell-Electrode Detector (CSED, Chinese Patent \#ZL201620361767.1). The detector concept of the CSED will be described in detail here. Full 3D simulations of the performance behavior of the CSED will be carried out and presented. These simulations include detector potential, electric field, and electron (or hole) concentration profiles, as well as detector leakage current, capacitance, and charge collection properties. Comprehensive comparisons between the CSED and the non-etch-through 3D-Trench electrode detectors will be made. The novel CSED has much better electric field profiles near the backside and are much better isolated from neighboring cells than that in non-etch-through 3D-Trench electrode detectors.
\end{abstract}

\section{Introduction}

Detectors based on semiconductor materials have been used in various fields such as deep space imaging, medical imaging (e.g., positron emission tomography), tracking in high-energy physics, radiation-trace detection for the purpose of national security, etc. In the LHC (Large Hadron Collider) and HL-LHC (High Luminosity-LHC) at CERN, for example, the total radiation fluence can reach up to $1 \times 1016 \mathrm{neq} / \mathrm{cm} 2[1]$. In such high radiation environment, high radiation will cause displacement damage in $\mathrm{Si}$, which in turn will increase the defect concentration in the detector. Effects of such defects will change detector electrical properties such as increase of detector leakage current and effective space charge, and reduction in detector charge collection efficiency. The interest for 3D column electrode silicon detectors is continuously growing because of their many advantages as compared to conventional planar detectors due to the separation of detector electrode spacing from the detector thickness. For these detectors, if the column spacing is made much smaller than the detector thickness, they can be fully depleted at much lower bias voltages and exhibit higher radiation tolerance, as compared to planar detectors. However, the disadvantage of such detectors is the distribution of non-uniform electric field and the existence of vast low-field region[2, 3].

To improve electric field distribution and charge collection, a new type of 3D detector: 3D-trench electrode detector has been proposed at BNL in 2009[4], as shown Fig.1[1, 2, 5 6]. The 3D-Trench Electrode detector not only can be radiation tolerant, but also it has more uniform electric field distribution as compared to the 3D column electrode detector. However, this 3D-Trench Electrode structure also has a deficiency: the trench electrode can't be fully etched through (or else the detector cell enclosed by the trench electrode will fall off the wafer). Although a support wafer or SOI technology may be used to overcome this deficiency[5], it will significantly increase the detector fabrication cost and complexity. The part of non-etching-through region (typically $10 \%$ of the detector thickness) gives rise to the low electric field region in the detector and provide an electronic connection between neighboring cells in the detector, thus compromising the detector charge collection efficiency and cell isolation. In order to overcome this deficiency in the non-etch-through 3D-Trench Electrode detector, a novel structure has been proposed in Xiangtan University (XTU) in 
2016. To separate it from the non-etch-through 3D-Trench Electrode detectors, we call it as the Closed Shell-Electrode Detector(CSED) (Chinese Patent \#ZL201620361767.1, Pub. Date: Aug. 31, 2016).

This article is mainly focused on the description of the CSED structure concept and 3D performance simulations, including 3D distributions of electric potential, field and hole (electron) concentration; detector electrical properties such as detector leakage current (I) and capacitance (C) as a function of bias voltages (V) (IV, CV); and detector full depletion voltage.

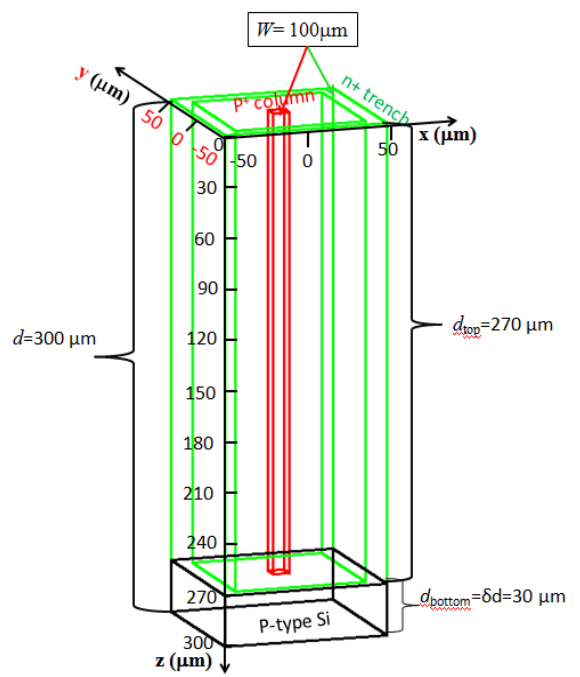

Fig.1 An example of the structure of a square 3D-Trench Electrode Silicon detector
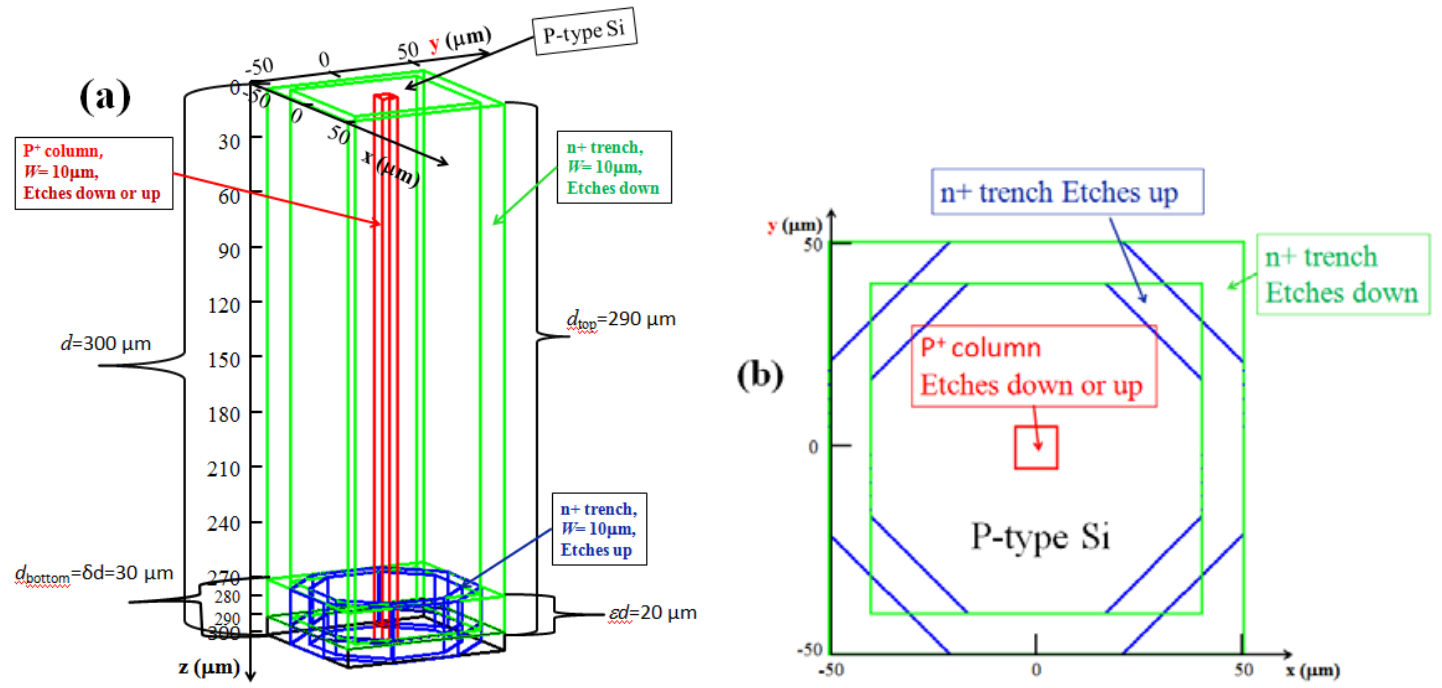

Fig.2 An example of the structure of a square shape novel CSED structure: a) the 3D view of the entire single cell, and $b)$ the cross section view $(x-y$ plane, at $z=270 \mu \mathrm{m})$ of the CSED in the portion of the interleaved trenches.

\section{Device Description and Simulation}

For comparison purposes, a single cell of a non-etch-through square 3D-Trench Electrode detector is shown Fig.1. The non-etch-through portion is clearly shown in the bottom of the cell, which typically occupies $6 \%-15 \%$ of the cell volume.

A single cell of a novel closed-shell electrode silicon detector with square shape is illustrated in Fig. 2. As shown Fig. 2a, this structure is composed of two different structures, square and octagonal, interleaved to each other. In our case shown here, the square trench is etched from the top side (from 
top down), but not etch through with a depth of dtop $<\mathrm{d}$, where $\mathrm{d}$ is the thickness of the detector (typically $\mathrm{d} \leq 500 \mu \mathrm{m}$ ). The octagonal trench is etched from the bottom side (from bottom up), again not etch through (with a depth of $\mathrm{dbott}=\delta \mathrm{d}, 0<\delta<1$ ) but has an overlap in the thickness direction with the square trench. The depth of overlap part is defined as $\varepsilon d$, where $0 \leq \varepsilon<<1$. As shown in Fig. $2 b$, the top and bottom trenches are disconnected in four corners. It is clear that we have the following relationship dtop $=(1-\delta+\varepsilon)$ d between various depths. The central column electrode can be etched all the way through the detector thickness, either from top down or bottom up depending on the specific processing need. The top-etched and bottom-etched trenches are doped the same way (in our case phosphorous or $\mathrm{n}^{+}$-type), and the central column is doped in opposed way (in our case boron or p+-type). In our example for simulations, Si bulk is chosen as p-type with a doping concentration of $1 \times 1012 \mathrm{~cm}-3$, the edge length of the trench electrode is set at $100 \mu \mathrm{m}$, the width of all trenches are set at $10 \mu \mathrm{m}$, the width of the square central column is set at $10 \mu \mathrm{m}, \mathrm{d}=30 \mu \mathrm{m}, \delta=0.1$, and $\varepsilon=0.067$.

Due to the arrangement of the top and bottom interleaved trenches, their enclosed cell bulk will not fall off the wafer, even though the overall trench electrode is effective "etch-through" with top and bottom trenches are connected in large areas (they are not connected in four corners) as shown in Fig. 2b. It is clear that, the larger the cell size, the bigger the connected portion of the top and bottom trenches. Neighboring cells are connected to each other and to the detector main body through the unconnected portion, and therefore will not fall off the wafer. The overlapping of the top and bottom trenches in the thickness direction ensures the electronic connection between the neighboring cells being minimum to achieving effective cell isolation.

Full 3D simulations on a Closed-Shell Electrode Silicon detector shown in Fig. 2 have been performed using the DEVICE 3D package of Silvaco's ATLAS. The $\mathrm{n}+$ outer trenches are doped with phosphorus of $1019 / \mathrm{cm} 3$, and the $\mathrm{p}+$ central column is doped with boron of $1019 / \mathrm{cm} 3$. The choice of p-type bulk is for the radiation hardness consideration since under the high radiation influence, n-type silicon will space charge sign invert to p-type like[6]. Here, the p-n junction is formed between the bulk and the outer ring trench (ORJ, or outer-ring-junction). Compared to the case where the junction is formed at the central column electrode (CJ, center-junction), detectors with ORJ have more uniform electric filed profile and much smaller maximum electric field and full depletion voltage [1,6]. From ref. [1], in fact one can write down the electric field profiles beyond full depletion for the perfect cylindrical geometry as the following:

$$
\begin{aligned}
& \left.E(r)=\frac{1}{2} \frac{e N_{e f f}}{\varepsilon \varepsilon_{0}} r\left[1-\frac{r_{c}^{2}}{r^{2}}\right]-\frac{V-V_{f d}}{r \ln R / r_{c}} \quad\left(r_{c} \leq r \leq R, O R J\right) \quad a\right) \\
& E(r)=\frac{1}{2} \frac{e N_{e f f}}{\varepsilon \varepsilon_{0}} r\left[\frac{R^{2}}{r^{2}}-1\right]+\frac{V-V_{f d}}{r \ln R / r_{c}} \quad\left(r_{c} \leq r \leq R, C J\right) \quad b
\end{aligned}
$$

Where Eq. (1)a is for the ORJ case and Eq. (1)b is for the CJ case, $V_{f d}$ is the full deplete voltage, Neff is the effect doping concentration, $\mathrm{R}$ is inner radius of outer trench (in this case, it is the radius of the fully depleted region), $r_{c}$ is the radius of central column electrode, $V_{b i}$ is the built-in potential, $\varepsilon_{0}$ is the vacuum dielectric constant, and $\varepsilon$ is the relative dielectric constant for silicon. We can use Eq. (1) to qualitatively estimate the electric filed profiles in our non-perfect square and octagonal cases before full 3D simulations. For example, at full depletion voltage $\mathrm{V}=\mathrm{V}_{\mathrm{fd}}$, the maximum electric field is at $r=R$ for the ORJ case at, which is much smaller than that for the CJ case at if $\mathrm{R}>>\mathrm{r}_{\mathrm{c}}$. In fact, for our case where $\mathrm{R}=50 \mu \mathrm{m}, \mathrm{r}_{\mathrm{c}}=10 \mu \mathrm{m}$, it is about 4 times smaller!

After full 3D simulations, in order to clearly illustrate resulting distributions inside the detector cell, we have to plot 2D cut planes along the different sections the structure shown in Fig. 1 and Fig. 2a. For comparison purposes, we plot in Fig. 3 a 2D cut plane of the electric profile of a 3D-Trench Electrode detector shown in Fig. 1. The bias voltage here is set as $1 \mathrm{~V}$. The cut plane is in the $\mathrm{x}-\mathrm{Z}$ plane $(\mathrm{y}=0 \mu \mathrm{m})$. Shown in Fig. 4 is the same for a Closed-Shell Electrode Silicon Detector shown in Fig. 2a. Comparing simulation results in Fig. 3 and Fig. 4 one can clearly see that: 1) Along the $z$ axis direction, the electric field profile is more uniform in the bottom portion (from $z=270 \mu \mathrm{m}$ to $300 \mu \mathrm{m})$ in the CSED than that in the non-etch-through 3D-Trench Electrode detector; 2) the electric 
field is much higher in the bottom portion of the CSED as well as compared to the low electric field in the bottom portion of 3D-Trench Electrode detectors; and 3) the cell is isolated from other cells where the trenches are connected in the CSED, while cells are connected by the bottom portion in the non-etch-through 3D-Trench Electrode detector. Therefore the novel CSED are much better than the non-etch-through 3D-Trench Electrode detector in these regards. Apparently CSED fabrication requires double-sided process, which is nowadays quite standard and routinely done in $3 \mathrm{D}$ detector fabrications [7, 8], Fig. 5 shows simulated electric field profiles in 2D cuts along the $x-y$ plane at two different $z$ positions the CSED shown in Fig. 2: $z=100 \mu \mathrm{m}$ (Fig. 5a) and $z=270 \mu \mathrm{m}$ (Fig. 5b). As shown Fig. 5a, high electric fields are near the outer square trenches (since the junction is near the trench electrode) with slight non-uniformity near the corners. In fact this electric filed profile should be no different from that in the not etched through 3D-Trench Electrode detectors at the same $\mathrm{z}$ position since they have the same geometry at this $\mathrm{z}$ position. However, as shown in Fig. 5b, at $\mathrm{z}=270 \mu \mathrm{m}$, where the top square trench and the bottom octagonal trench are overlapped, the electric filed profile is quite different from that shown in Fig. 5a. First, the electric field profile in the bottom portion is more uniform since the octagonal shape is closer to the circle than the square shape; and 2) there exist four triangular regions near the square trench corners that are effectively cut off from the detector sensitive region, and can be considered as the dead region. We will discuss the percentage of this dead region as a function of the detector geometry in more details in the next section.

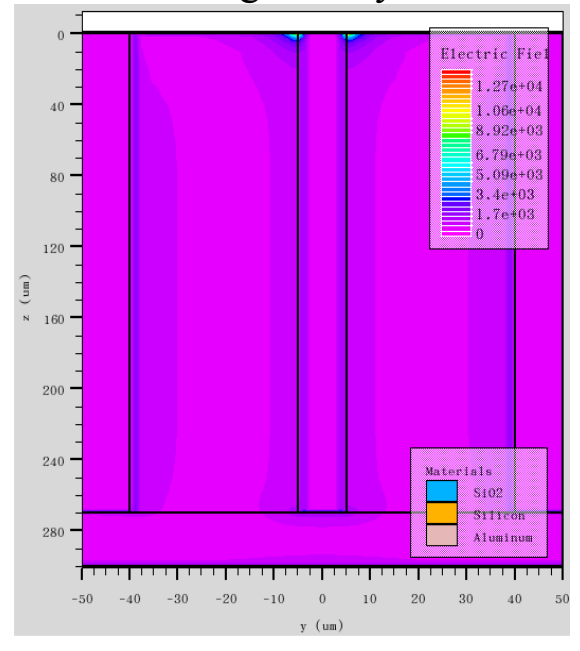

Fig. 3 Simulated electric field profile along a $2 \mathrm{D}$ cut plane on the $\mathrm{y}-\mathrm{z}$ plane at $\mathrm{x}=0$ for a non-etch-through 3D-Trench Electrode detector (see Fig. 1).

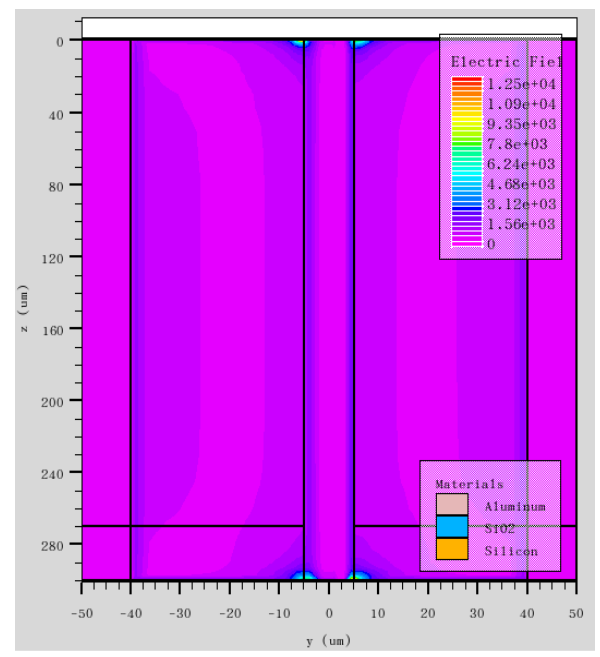

Fig. 4 Simulated electric field profile along a $2 \mathrm{D}$ cut plane on the $\mathrm{y}-\mathrm{z}$ plane at $\mathrm{x}=0$ for a CSED (see Fig. 2a). 
(a)

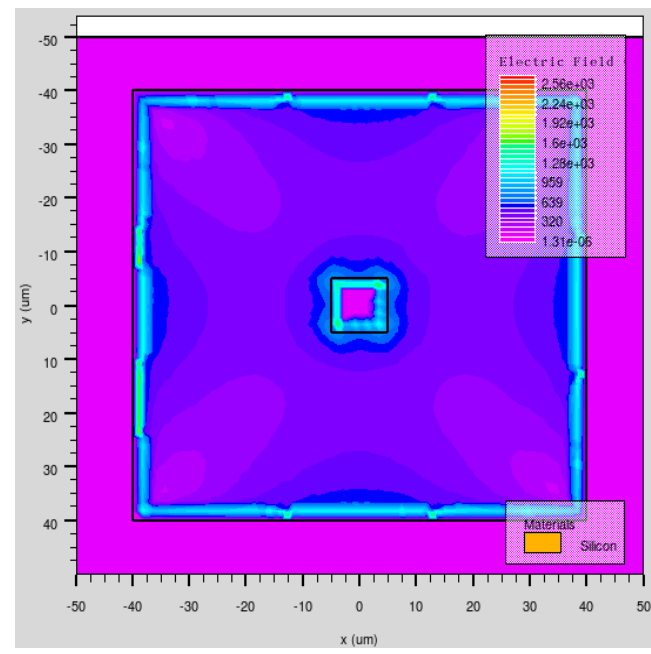

(b)

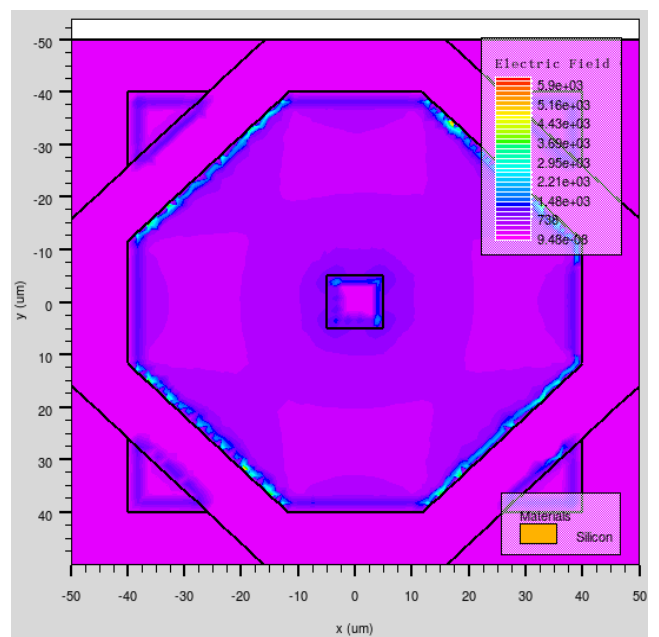

Fig. 5 Simulated electric field profile for a CSED (see Fig. 2a): a) along a 2D cut plane on the x-y plane at $\mathrm{z}=100 \mu \mathrm{m}$; and $\mathrm{b}$ ) along a $2 \mathrm{D}$ cut plane on the $\mathrm{x}-\mathrm{y}$ plane at $\mathrm{z}=270 \mu \mathrm{m}$.

Fig. 6 shows the simulated detector leakage current as a function of the bias voltage (I-V curve) for CSED and not etched through 3D-Trench Electrode detectors. Detector shown in Fig. 6a is the same CSED shown previously in Fig. 2. We can see from the I-V curve that the detector is fully depleted near 1 volt, which is very close to the value calculated using equations in ref. [1] for the cylindrical geometry. Also, the breakdown voltage for this particular CSED is about 174 volt, giving a large detector work range ( 1 volt $<\mathrm{V}<174$ volt). For comparison purposes, a simulated $\mathrm{I}-\mathrm{V}$ curve for a 3D-Trench Electrode detector (see Fig. 1) is shown in Fig. 6b. It is clear that the full depletion voltage for a CSED is smaller than that for a 3D-Trench Electrode detector with similar geometry. Also the CSED shows a slightly higher breakdown voltage.
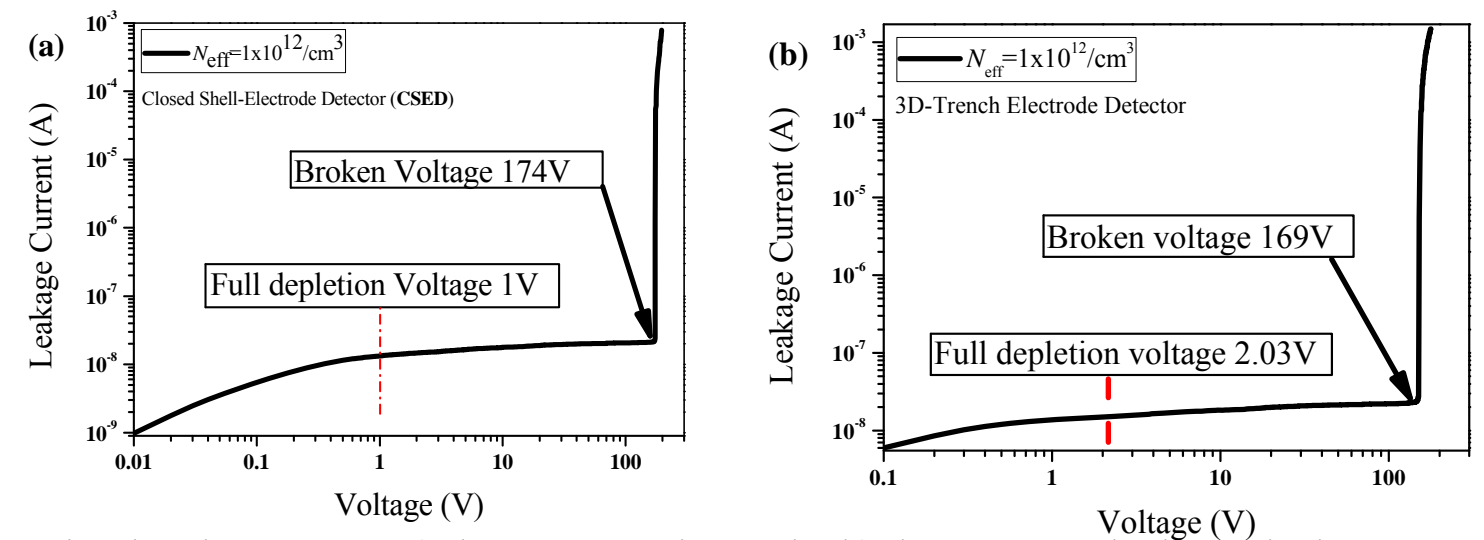

Fig. 6 Simulated I-V curve: a) the CSED under study, b) the 3D-Trench electrode detector under study.

\section{Full Depletion Voltage}

As we know, the full depletion voltage of a planar detector is related to the detector thickness. For all types of 3D detectors, however, the detector full deplete voltage depends only on the electrode spacing $[9,10]$. For the 3D-Trench Electrode detector, the detector full depletion voltage has been calculated for cylindrical geometry for the ORJ and CJ cases [1]:

$$
\begin{aligned}
& \left.V_{f d}=\frac{e N_{e f f}}{2 \varepsilon \varepsilon_{0}}\left[\frac{1}{2}\left(R^{2}-r_{c}^{2}\right)-r_{c}^{2} \ln \frac{R}{r_{c}}\right]-V_{b i}(O R J) \quad a\right) \\
& \left.V_{f d}=\frac{e N_{e f f}}{2 \varepsilon \varepsilon_{0}}\left[R^{2} \ln \frac{R}{r_{c}}-\frac{1}{2}\left(R^{2}-r_{c}^{2}\right)\right]-V_{b i}(C J) \quad b\right\}
\end{aligned}
$$


Where Eq. (2)a is for the ORJ case and Eq. (2)b is for the CJ case.

In this paper, the detector full depletion voltage of the novel CSED has been extracted from the 3D simulation of electric field profiles. Fig. 7 shows under different bias voltages the electric field distribution with radius along different 1D cut lines in Fig. 5. Shown in Fig. 7a are 1D electric field profiles along radius from $(0,0)$ to $(50,0)$ in the CSED shown in Fig. 5, and shown in Fig. $7 \mathrm{~b}$ are those along radius from $(0,0)$ to $(50,50)$ for the same CSED. It is clear that there exist electric field in the entire region between the trench electrode and central column electrode at a bias voltage of 1 volt in both cased, indicating the detector is fully depleted at $\mathrm{V}=1$ volt. This is in agreement with our previous estimations from calculation using Eq. $3 \mathrm{~b}$ and I-V curve in Fig. 6.

We notice here that volumes between the top and bottom trenches where they are not connected are in fact cut off from the detector sensitive volume, and therefore are dead space. One can easily calculate the percentage portion of this dead space in a single cell for different cell geometries. It is apparent that it will decrease as the cell size increases. It also depends on the depths of the top and bottom trenches and their overlap $(\varepsilon)$. Details of dead volume study will be reported elsewhere in a different report. For our particular case shown in Fig. 5, it is only about $0.65 \%$, which is nearly negligible.
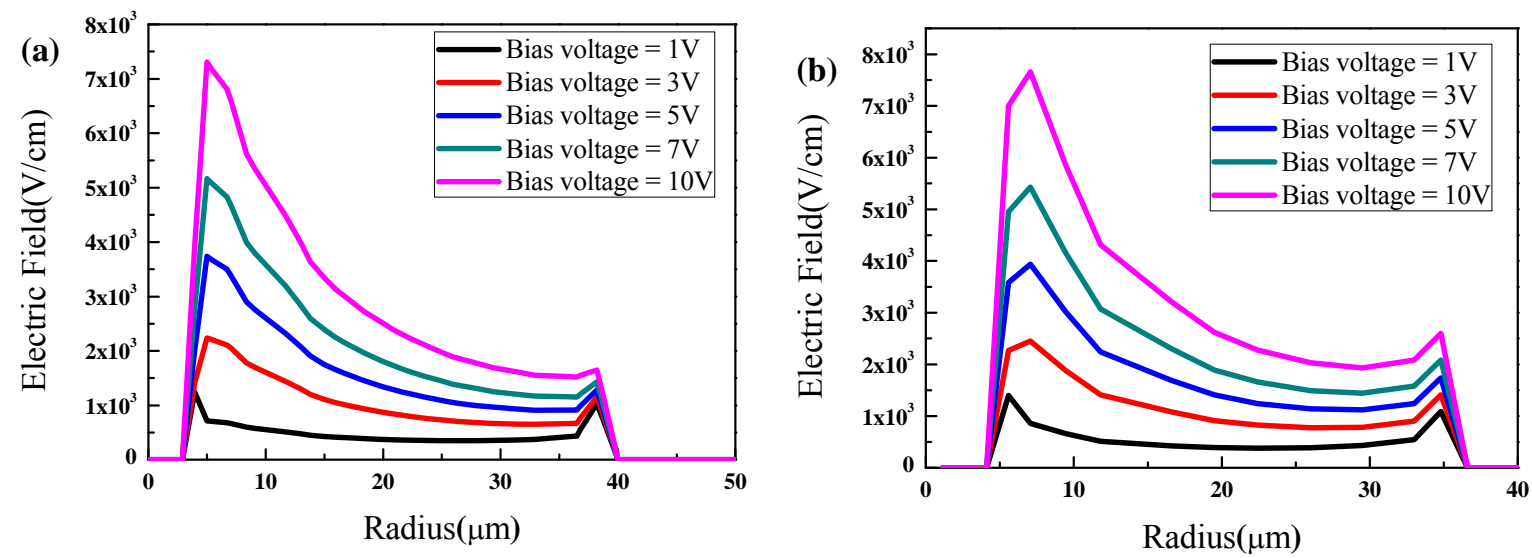

Fig.7 Electric field distribution under different bias voltages,

a) along cutline from $(0,0)$ to $(50,0)$ and b) along cutline from $(0,0)$ to $(50,50)$ in Fig. 5 .

\section{Potential Characteristics}

The potential profile for this particular CSED structure is shown in Fig. 5 are also simulated using a the DEVICE 3D package of Silvaco's ATLAS simulation tool. Simulated electric potential profile is shown in Fig. 8 in a 3D way, which is along a 2D plane at $\mathrm{z}=100 \mathrm{~m}$. The bias voltage is 1volt. It is clear that, the potential distribution is uniform without any saddle points as in the case of 3D column electrode detector [11].

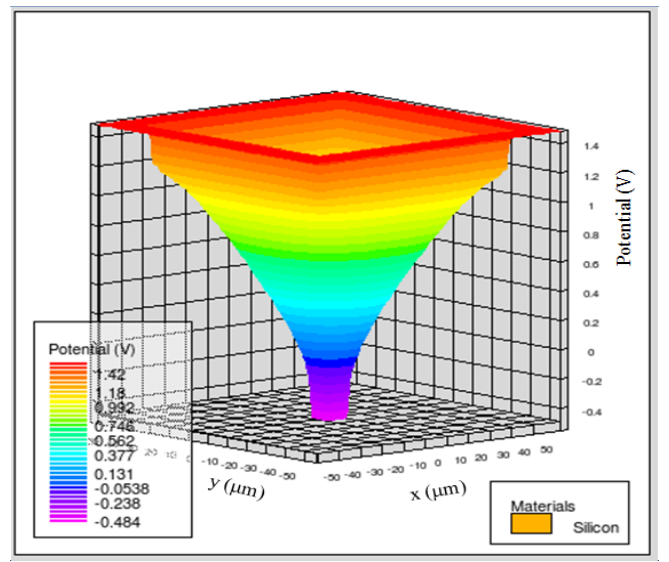

Fig. 8 Simulated electric potential profile for the CSED sstuied here. The 2D cut plane is at $\mathrm{z}=100 \mu \mathrm{m}$. 


\section{Capacitance- Voltage Characteristics}

Capacitance is an important parameter for a detector, since the detector noise goes up with the detector capacitance. The capacitance of a fully depleted square CSED can be approximated theoretically by using the capacitance of a cylindrical capacitor $[5,12,13]$ :

$$
C_{c y l}=2 \pi \varepsilon_{r} \varepsilon_{0} \frac{d}{\ln \left(R / r_{c}\right)}
$$

where $\mathrm{C}_{\mathrm{cyl}}$ is the capacitance of the CSED.

Fig. 9 is the simulated C-V curve for the CSED shown in Fig. 5.

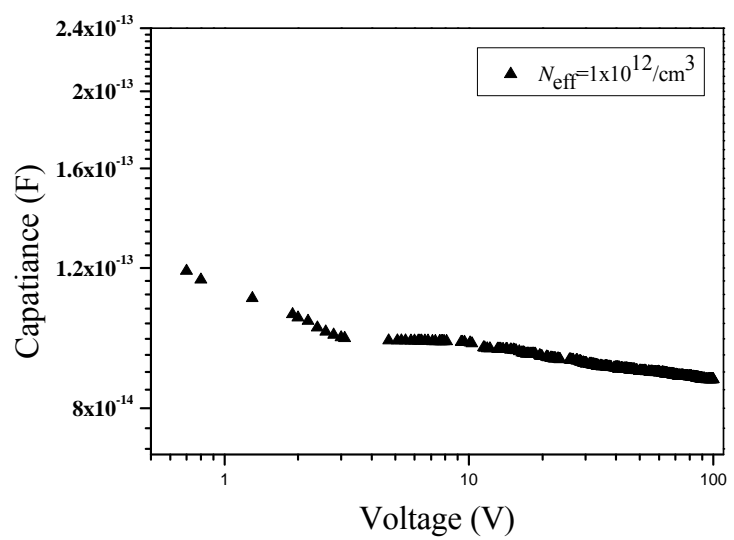

Fig.9 C-V characteristics without radiation

As shown Fig.9, it is clear that the detector capacitance decreases with the bias voltage and becomes flat (reaching the geometry capacitance) around 2-3 volts, which is larger than that for a flat $\mathrm{I}-\mathrm{V}$ curve and has been routinely observed before for planar detectors. The geometry capacitance is about $100 \mathrm{fF}$, which is close to calculated capacitance using Eq. 4.

\section{Conclusion}

A novel Closed-Shell-Electrode-Detector (CSED) has been proposed and described in details. Full 3D simulations have been conducted using a 3D-TCAD tool on the CSED, including electric potential and field profiles, detector leakage current, and detector capacitance. Compared to the non-etch-through 3D-Trench electrode detectors, the electric filed profile in the bottom portion (the non-etched region in the non-etch-through 3D-Trench electrode detectors and the bottom trench region in the CSED) is more uniform and much larger in the CSED, and cells are better separated in the CSED. The dead space is very small (only $0.65 \%$ in our case here in this study), and can be made even smaller by increasing the cell size. It requires a double-sided fabrication processing, which is quite standard in 3D detector fabrications.

\section{References}

[1] Z. Li, Nucl. Instrum. Methods Phys. Res., Sect. A, 658 (2011) 90-97.

[2] T. Grönlund, et al., Nucl. Instrum. Methods Phys. Res., Sect. A, 586 (2008) 180-189.

[3] Z. Li, T. Grönlund, Nucl. Instrum. Methods Phys. Res., Sect. A, 612 (2010) 509-515.

[4] Z. Li, New detectors with novel electrode configurations for applications in sLHC and photon sciences, presented at the Fifteenth Workshop of CERN RD50, CERN, Geneva, Switzerland, 16-18 November, 2009.

[5] H. Ding, et al., Nucl. Instrum. Methods Phys. Res., Sect. A 796 (2015) 29-33.

[6] A. Montalbano, et al., Nucl. Instrum. Methods Phys. Res., Sect. A, 765 (2014) 23-28. 
[7] A. Zoboli, et. al., IEEE TNS 55-5 (2008), p. 2775.

[8] G. Pellegrini, et al., Nucl. Instrum. Methods Phys. Res., Sect. A, 592 (2008) 38-43.

[9] J. Kalliopuska, S. Eränen, R. Orava, Nucl. Instrum. Methods Phys. Res., Sect. A, 568 (2006) $27-33$

[10] S.I. Parkera, C.J. Kenneya, J. Segalb, Nucl. Instrum. Methods Phys. Res., Sect. A, 395 (1997) 328-343.

[11] C. Piemonte, et al., Nucl. Instrum. Methods Phys. Res., Sect. A, 541 (2005) 441-448.

[12] J. Chen, et al., Nucl. Instrum. Methods Phys. Res., Sect. A, 796 (2015) 34-37.

[13] D.K. Schroder, T.T. Braggins, H.M. Hobgood, Journal of Applied Physics, 49 (1978) 5256. 